\title{
Estudio de la resistencia a tracción en anclajes estructurales posinstalados con adhesivo epóxico*
}

\author{
Diego Fernando Páez Moreno* \\ Jeniffer Hamón Caicedo**
}

Recibido: 29/06/2016 • Aceptado: 04/03/2018

https://doi.org/10.22395/rium.v17n33a3

\begin{abstract}
Resumen
Investigaciones realizadas en anclajes estructurales posinstalados, han evidenciado un vínculo entre la longitud de embebido y el diámetro de perforación con la resistencia máxima a tracción. El presente artículo, resultado de una investigación financiada por la dirección de investigaciones de la Universidad Pedagógica y Tecnológica de Colombia, analiza este vínculo a partir de la variación de la resistencia y el tipo de falla en prototipos de anclaje estructural con variaciones en la longitud de embebido y diámetro de perforación, en concreto de resistencia a la compresión $28 \mathrm{MPa}$, adhesivo epóxico, barra de refuerzo, 15 unidades de grafil de diámetro $0,007 \mathrm{~m}$, y 15 unidades de acero corrugado de diámetro 0,0095 m, para un total de 30 sistemas solicitados a tracción directa en la máquina universal. Los resultados mostraron que la resistencia a tracción y el tipo de falla están en función de la longitud de embebido y la estructura corrugada de la barra de refuerzo anclada.
\end{abstract}

Palabras clave: resina epóxica; anclajes adhesivos; resistencia.

* Artículo original derivado de investigación terminada financiada por la Dirección de Investigaciones de la Universidad Pedagógica y Tecnológica de Colombia.

** Magíster en Ingeniería Civil. Ingeniero Civil. Profesor investigador de la Universidad Pedagógica y Tecnológica de Colombia, Facultad de Ingeniería, grupo de investigación Grinfavial. Avenida Central del Norte N. ${ }^{\circ} 39-115$. Teléfono: +57+8 7405626. Correo electrónico: diego.paez@uptc.edu.co. Orcid:http://orcid.org/0000-0003-1141-8819.

*** Ingeniera civil, estudiante de Maestría en Ingeniería Civil, miembro del grupo de investigación Grinfavial de la Universidad Pedagógica y Tecnológica de Colombia. Avenida Central del Norte N. ${ }^{\circ} 39-115$. Teléfono: $+57+8$ 7405626. Correo electrónico: jennifer.hamoncaicedo@uptc.edu.co. Orcid: https://orcid.org/0000-0003-3013-5611. 


\title{
Study of Tensile Strength in Structural Anchors Post-Installed with Epoxy Adhesive
}

\begin{abstract}
Research carried out on post-installed structural anchors has shown a link between the embedded length and the diameter of the bore with maximum tensile strength. This paper, is the outcome of research funded by the research department of the Universidad Pedagógica y Tecnológica de Colombia, analyzes this link from the variation of resistance and failure type in structural anchor prototypes with variations in embedded length and drilling diameter, in particular compressive strength $28 \mathrm{MPa}$, epoxy adhesive, reinforcing bar, 15 graphite units of $0.007 \mathrm{~m}$ diameter, and 15 corrugated steel units of $0.0095 \mathrm{~m}$ diameter, for a total of 30 systems requested for direct traction in the universal machine. The results showed that tensile strength and failure type are a function of the embedded length and corrugated structure of the anchored reinforcing bar.
\end{abstract}

Keywords: epoxy resin; adhesive anchors; strength.

\section{Estudo da resistência à tração em ancoragens estruturais pós-instaladas com adesivo epóxi}

\begin{abstract}
Resumo
Pesquisas realizadas em ancoragens estruturais pós-instaladas evidenciaram um vínculo entre o comprimento de incorporação e o diâmetro de perfuração com a resistência máxima à tração. $\mathrm{O}$ presente artigo, resultado de uma pesquisa financiada pela direção de pesquisas da Universidad Pedagógica y Tecnológica de Colombia, analisa esse vínculo a partir da variação da resistência e do tipo de falha em protótipos de ancoragem estrutural com variações no comprimento de incorporação e diâmetro de perfuração em concreto de resistência à compressão $28 \mathrm{MPa}$, adesivo epóxi, barra de reforço, 15 unidades de arame trefilado de 0,007 $\mathrm{m}$ de diâmetro e 15 unidades de aço corrugado de 0,0095 m de diâmetro, para um total de 30 sistemas solicitados à tração direta na máquina universal. Os resultados mostraram que a resistência à tração e ao tipo de falha ocorrem em função do comprimento de incorporação e da estrutura corrugada da barra de reforço ancorada.
\end{abstract}

Palavras-chave: resina epóxi; ancoragens adesivas; resistência. 


\section{INTRODUCCIÓN}

En Colombia existe una cantidad importante de edificaciones construidas antes de la entrada en vigencia de documentos técnicos como el Reglamento colombiano de construcción sismo resistente NSR-10 [1], y por ello se justifica la necesidad de reforzar y restaurar este tipo de edificaciones con el fin de actualizarlas respecto a los nuevos requisitos de funcionalidad y servicio. Entre los métodos constructivos para el reforzamiento y la restauración de estructuras se encuentran los anclajes estructurales posinstalados con adhesivo epóxico [2].

Como lo especifica el Committee ACI 503 [3], dichos anclajes consisten en barras roscadas o conformadas que se cementan utilizando compuestos químicos de dos componentes formados. Estos se cargan principalmente a través de dispositivos de fijación del anclaje embebido y la solicitación puede ser de flexión, tracción, corte o una combinación de las anteriores. Investigaciones analíticas y experimentales se han desarrollado en esta temática con el fin de aportar claridad respecto a la interacción de las variables que actúan en los anclajes con adhesivo epóxico y la influencia que estas tienen en su comportamiento mecánico. Entre las analíticas se destacan varias. Una de ellas es la elaborada por Gesoğlu et al. [4] en la cual, con el uso de programación de expresión de genes (GEP), se desarrolló un modelo de predicción de la capacidad de cortante en anclajes posinstalados en concreto endurecido. Otra es expuesta por Obataa et al. [5], que estudió experimental y analíticamente la influencia del borde libre en la resistencia a la tracción de los anclajes exhibiendo una relación entre la longitud de embebido y la falla. Finalmente, la de Upadhyaya y Kumar [6] hizo énfasis en predecir el esfuerzo que causaría la falla por cortante o tracción en anclajes estructurales posinstalados, determinando que, con el aumento en la longitud de embebido, se reduce el esfuerzo máximo a cortante.

Respecto a las experimentales, la investigación realizada por Çalıskan et al. [7] evaluaron anclajes estructurales con concreto de baja resistencia a compresión solicitados a cortante y concluyen que, al aumentar el diámetro del anclaje, se disminuye su resistencia a cortante. Yang et al. [8] por su parte, analizaron el arrancamiento del anclaje en un sistema compuesto por anclaje-mortero-concreto estableciendo que la carga máxima se incrementa linealmente con la longitud de embebido. En cuanto a los anclajes estructurales con concreto de alta resistencia, la investigación de Çolak [9] se enfocó principalmente en el comportamiento del adhesivo epóxido, señala la importancia en la dosificación de este e indica que, en este tipo de anclajes, no se obtienen mejores resultados con una profundidad de embebido mayor a 10 veces el diámetro de la barra.

Ruda y Páez [10] por su parte, evaluaron la resistencia a tracción directa en anclajes con dos tipos de barra refuerzo y concretos de alta resistencia, evidenciando 
que con una longitud de embebido de diez veces el diámetro de la barra de refuerzo es constante el desarrollo de la fluencia del acero, y que para este tipo de sistemas de anclaje es más recomendable emplear diámetros menores de acero de refuerzo. McVay [11] analizó las características propias del proceso de falla determinando que, en el concreto, el tipo de falla "cono" se debe a la tracción, mientras que la falla en la interface de concreto-adhesivo se debe al corte.

A partir de las investigaciones referenciadas, se evidencia la clara la influencia de la longitud de embebido y diámetro de perforación en el comportamiento de los anclajes estructurales posinstalados.

Otras investigaciones, como la desarrollada por Contrafatto y Cosenza [12] y Puigvert et al. [13], demostraron la posibilidad de emplear estos sistemas estructurales en basalto, arenisca y caliza al igual que nuevas aplicaciones como el reemplazo de las barras de refuerzo corrugadas por CFRP. Respecto al proceso constructivo de los anclajes estructurales posinstalados, el documento elaborado por Gerber y Ekel [14] expone el contraste entre los requisitos establecidos por diferentes estándares para la inspección de anclajes estructurales posinstalados. Adicionalmente, documentos técnicos como el de Hilti [15] compilan lineamientos a considerar en cuanto a directrices de diseño e instalación de anclajes estructurales. Particularmente, en este documento se incluye un capítulo con la descripción de las ecuaciones de diseño congregadas en el Commitee ACI 318-11 [16] para establecer la longitud de embebido (empotramiento) en anclajes, sin embargo, en las hojas técnicas de fabricantes de adhesivo epóxico como lo es SIKA [17] se establece un valor específico para la longitud de embebido y diámetro de perforación según el diámetro de la barra de refuerzo.

Con base en el contexto descrito previamente, y dada la necesidad de continuar evaluando la influencia que tienen las variables de diámetro de perforación y longitud de embebido sobre los anclajes estructurales posinstalados, se estructura la presente investigación con anclajes estructurales posinstalados solicitados a tracción directa, construidos a partir de probetas de concreto con un tipo de resistencia a la compresión y dos tipos de barra de refuerzo. De esta forma, los parámetros a evaluar fueron la carga última resistida por cada sistema de anclaje estructural y su respectivo tipo de falla. Cabe destacar que esta investigación no considera las condiciones de confinamiento del concreto reforzado.

\section{MATERIALES Y MÉTODOS}

Con énfasis en los métodos y materiales constructivos regionales, se emplearon probetas de concreto con un tipo de resistencia a la compresión comúnmente empleada en el sector de la construcción [18], barras de refuerzo con dos diámetros nominales diferentes, y adhesivo epóxico Sikadur ${ }^{\circledR}$ AnchorFix-4 de Sika S. A. 


\subsection{Concreto}

Se usaron 30 probetas cilíndricas de concreto para desarrollar los ensayos de resistencia de los anclajes estructurales posinstalados y 16 probetas para evaluar la resistencia a la compresión a los 28 días de edad. Se estableció la resistencia máxima a compresión promedio en 28 MPa según la NTC 673 [19], asimismo, se estableció la homogeneidad de este parámetro respecto a la media aritmética establecida con base en los datos dados por los proveedores. La caracterización del concreto se muestra en la tabla 1.

Tabla 1. Características elementos de anclaje estructural posinstalado

\begin{tabular}{|c|c|c|c|c|c|c|c|}
\hline \multicolumn{5}{|c|}{ Características fisicas } & \multicolumn{3}{|c|}{ Características mecánicas } \\
\hline \multicolumn{2}{|c|}{$\begin{array}{c}\text { Material } \\
\varnothing \\
\end{array}$} & \multicolumn{2}{|c|}{$\begin{array}{l}\text { Dimensiones } \\
\text { (m) Longitud }\end{array}$} & \multirow{2}{*}{$\frac{\text { Cantidad }}{27}$} & \multirow{3}{*}{$\begin{array}{l}\text { Parámetro } \\
\text { fabricante } \\
\text { Resistencia efectiva a la } \\
\text { compresión }\end{array}$} & \multicolumn{2}{|c|}{$\begin{array}{c}\text { Fuente } \\
\text { laboratorio }\end{array}$} \\
\hline \multirow{2}{*}{ Concreto } & Lote 1 & \multirow{2}{*}{0,15} & \multirow{2}{*}{0,3} & & & $28,95 \mathrm{MPa}$ & $31,33 \mathrm{MPa}$ \\
\hline & Lote 2 & & & 27 & & $25 \mathrm{MPa}$ & $33,92 \mathrm{MPa}$ \\
\hline \multirow{5}{*}{$\begin{array}{l}\text { Barra de } \\
\text { refuerzo }\end{array}$} & \multirow{3}{*}{$\begin{array}{c}\text { Acero } \\
\text { corrugado }\end{array}$} & \multirow{3}{*}{0,009} & \multirow{3}{*}{0,15} & \multirow{3}{*}{22} & Carga rotura última & - & $35,2 \mathrm{kN}$ \\
\hline & & & & & Carga fluencia & - & $26,4 \mathrm{kN}$ \\
\hline & & & & & Resistencia fluencia & $420 \mathrm{MPa}$ & - \\
\hline & \multirow{2}{*}{ Grafil } & \multirow{2}{*}{0,007} & \multirow{2}{*}{0,15} & \multirow[t]{2}{*}{20} & $\begin{array}{l}\text { Resistencia mínima a la } \\
\text { tracción }\end{array}$ & $585 \mathrm{MPa}$ & - \\
\hline & & & & & Resistencia fluencia & $485 \mathrm{MPa}$ & - \\
\hline
\end{tabular}

Fuente: elaboración propia

\subsection{Barra de refuerzo}

Para los ensayos de tracción directa, se emplearon barras de refuerzo de diámetro $7 \mathrm{~mm}$ tipo grafil con características mecánicas dadas por el fabricante. También se utilizaron barras de refuerzo de diámetro 3/8" $(9,5 \mathrm{~mm})$ tipo acero corrugado Grado 60. De estas últimas se ensayaron dos unidades, con base en la NTC 2 [20] y se obtuvo su carga última de rotura, fluencia y la gráfica carga vs. alargamiento. La caracterización de las barras de refuerzo se muestra en la tabla 1.

\subsection{Adhesivo epóxico}

El adhesivo empleado es un sistema de tipo epóxico de dos componentes, $100 \%$ sólidos. Una vez mezclados los componentes, se obtiene una pasta suave de gran adherencia y resistencia mecánica para anclajes estructurales posinstalados. Cumple los requerimientos de la norma ASTM C-881-99, tipo IV, grado 3 [17]. Las características mecánicas principales del adhesivo epóxico utilizado se presentan en la tabla 2. 
Tabla 2. Principales características mecánicas adhesivo epóxico

\begin{tabular}{|l|c|}
\hline \multicolumn{2}{|c|}{ Características adhesivo epóxico } \\
\hline \multicolumn{1}{|c|}{ Parámetro } & Resistencia \\
\hline Tensión & $29,7 \mathrm{MPa}$ \\
\hline Corte & $25,5 \mathrm{MPa}$ \\
\hline Módulo de rotura & $46,2 \mathrm{MPa}$ \\
\hline
\end{tabular}

Fuente: elaboración propia

\section{PROCEDIMIENTO EXPERIMENTAL}

\subsection{Diseño experimental}

El diseño experimental empleado se ilustra en la figura 1 y se basa, tanto en la variación de la longitud de embebido (Le) definida como n veces el diámetro de la barra de refuerzo a anclar, como en la variación del diámetro de perforación (ØР) en el que se establecen tres medidas (bajo ØB, medio ØM, y alto ØА).

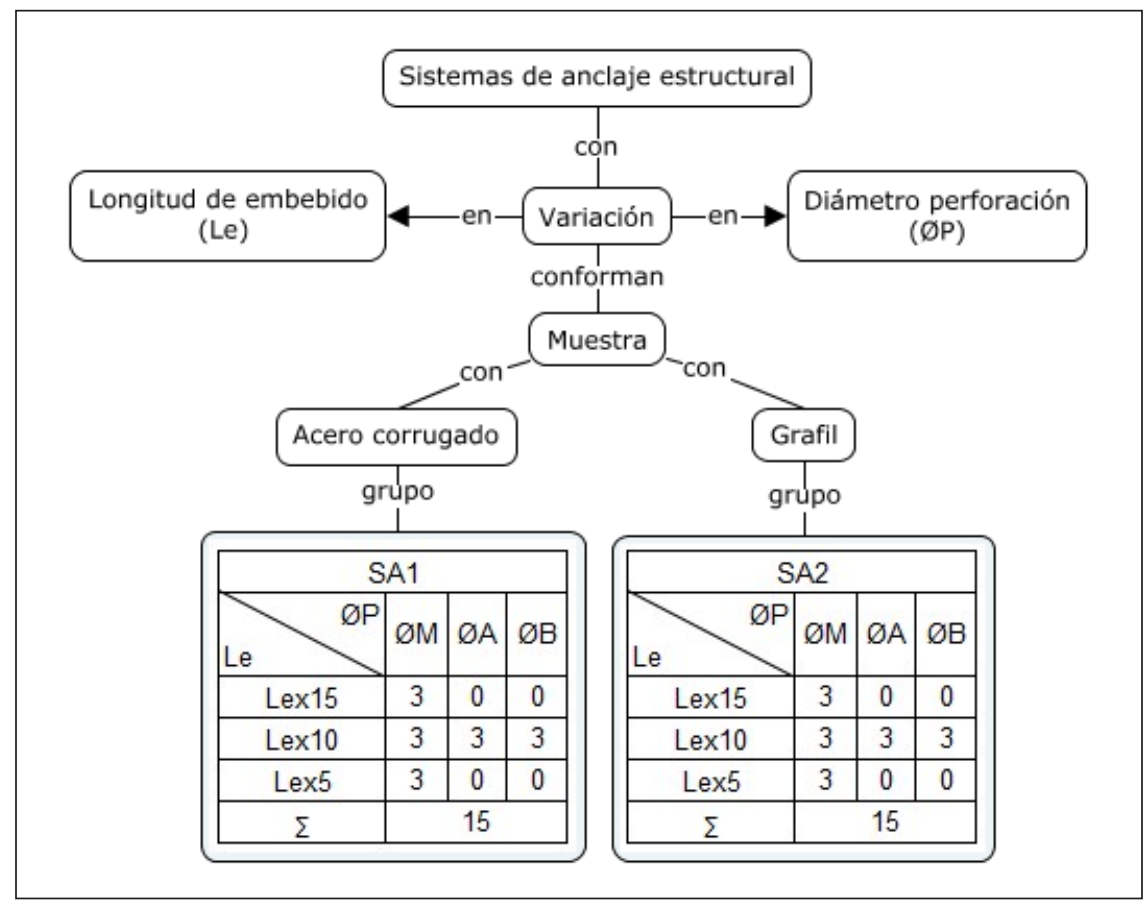

Figura 1. Diseño experimental para los ensayos en anclaje estructural posinstalado Fuente: elaboración propia 
En el caso de los anclajes estructurales posinstalados con grafil, a continuación, se especifica la equivalencia en el diámetro de perforación: $\varnothing \mathrm{B}=7,93 \mathrm{~mm} ; \varnothing \mathrm{M}=9,52$ $\mathrm{mm}$ y $\varnothing \mathrm{A}=12,7 \mathrm{~mm}$, y la equivalencia en la longitud de embebido (Le): $\varnothing \times 5=3,5$ $\mathrm{mm} ; \varnothing \times 10=7,0 \mathrm{~mm} \varnothing \times 15=10,5 \mathrm{~mm}$. Por otra parte, para los anclajes estructurales posinstalados con acero corrugado la equivalencia en el diámetro de perforación es: $\varnothing \mathrm{B}=9,52 \mathrm{~mm} ; \varnothing \mathrm{M}=12,7 \mathrm{~mm}$ y $\varnothing \mathrm{A}=15,8 \mathrm{~mm}$, y la equivalencia en la longitud de embebido (Le) es: $\varnothing \times 5=4,7 \mathrm{~mm} ; \varnothing \times 10=9,5 \mathrm{~mm} \emptyset \times 15=14,2 \mathrm{~mm}$. Cabe destacar que, para los anclajes con acero corrugado, el diámetro de perforación ØM y la longitud de embebido Øx10 son las especificadas por el fabricante del adhesivo epóxico [17].

\subsection{Preparación de los especímenes para el ensayo}

Para el desarrollo de los anclajes estructurales posinstalados se tuvieron en cuenta las instrucciones del fabricante del epóxico [17], siguiendo el orden que se enuncia:

- Perforación de probetas de concreto (pasado los 28 días de edad).

- Limpieza de la perforación con aire a presión.

- Aplicación del adhesivo epóxico mezclado en la boquilla hasta aproximadamente la mitad de la perforación.

- Inserción de la barra de refuerzo mediante movimientos rotativos.

- Curado de dos días.

\subsection{Ensayo a tracción directa}

Se procedió a someter los 30 anclajes estructurales posinstalados a un ensayo a tracción directa en máquina universal (como se muestra en la figura 2), con base en los lineamientos de ASTM E488M [21] para garantizar la estabilidad del anclaje estructural a ensayar, un área libre para el desarrollo de la falla, y que las fuerzas que fueran aplicadas a través de la barra de refuerzo fueran perpendiculares a la superficie de la sección del elemento de ensayo. Se empleó en la máquina universal un complemento de un armazón laminar y una platina de acero con un orificio circular dispuesto en el centro geométrico de 0,10 m como se muestra en la figura 3. La velocidad de carga empleada en el ensayo fue de $5 \mathrm{MPa} / \mathrm{s}$ de manera constante hasta llegar al punto de carga última del elemento. Inmediatamente finalizado el ensayo se procedió a tomar registro fílmico y fotográfico del tipo de falla presentado para su posterior caracterización según Committee ACI 355 [22]. 


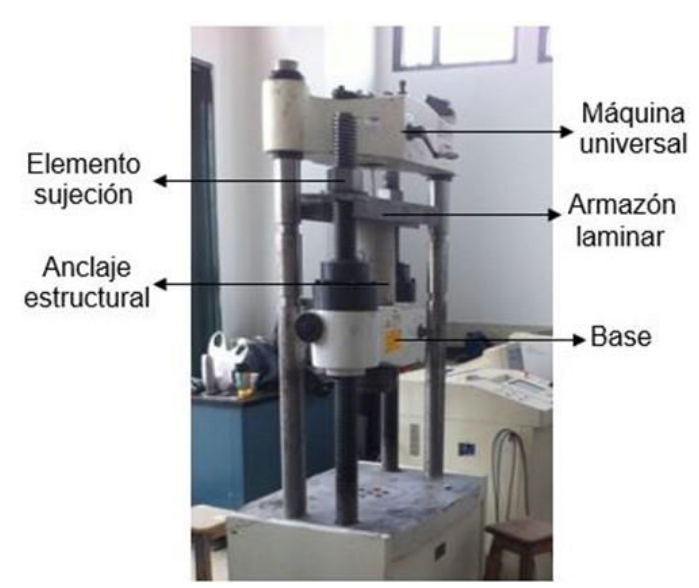

Figura 2. Montaje en máquina universal para ensayo a tracción directa

en anclaje estructural post instalado

Fuente: elaboración propia

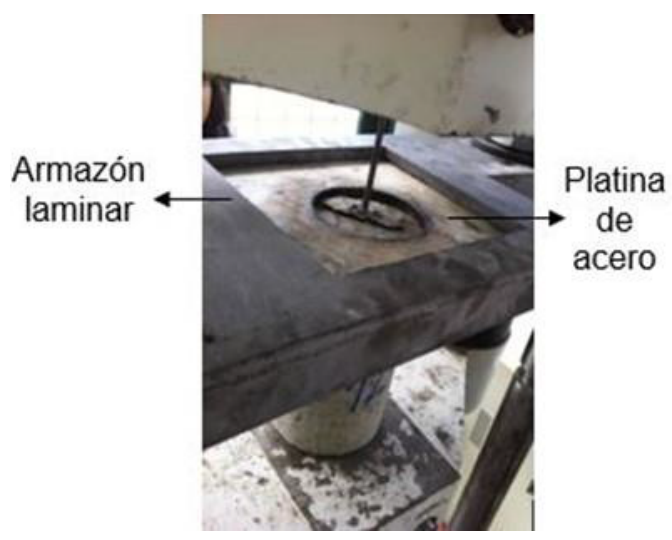

Figura 3. Soporte laminar y platina de acero para ensayo a tracción directa

en anclaje estructural posinstalado

Fuente: elaboración propia

\section{RESULTADOS}

El análisis se basa en una evaluación cuantitativa y una cualitativa de los resultados. La cualitativa consiste en la caracterización del tipo de falla clasificándola en falla por arrancamiento, falla del acero o falla por rotura del concreto. En contraste, la cuantitativa se basa en la premisa de que, en el desarrollo de los anclajes estructurales posinstalados, la fluencia de la barra anteceda la falla del sistema. Para este análisis de los resultados se tuvieron como referencia los valores de carga de fluencia, carga última y carga mínima de la barra de acero corrugado y el grafil. Con esto, se desarrollaron 
dos tipos de gráficas: una de tipo columna que exhibe la carga máxima soportada por cada anclaje adhesivo incluyendo líneas que representan los parámetros de carga mencionados según el caso, y otra de tipo línea que muestra las curvas de resistencia en función de la carga $(\mathrm{kN})$ vs. alargamiento $(\mathrm{mm})$.

Para el grupo de anclajes estructurales posinstalados con acero corrugado de diámetro 3/8" (9,5 $\mathrm{mm})$, el análisis cualitativo permitió establecer que, en la mayoría de anclajes la falla se dio por rotura del concreto, como se muestra en la figura 4. En contraste, se destaca que el tipo de falla presentado en los anclajes con mayor longitud de embebido (Lex15) fue por acero, lo cual se muestra en la figura 5. Esta falla está caracterizada porque la longitud embebida del anclaje fue suficiente para que la resistencia del acero controlara la rotura excluyendo así las fallas del concreto.

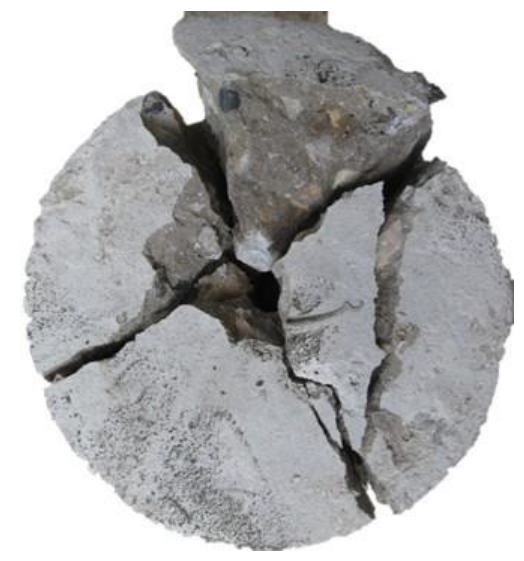

Figura 4. Falla por rotura del concreto en anclaje estructural posinstalado Fuente: elaboración propia

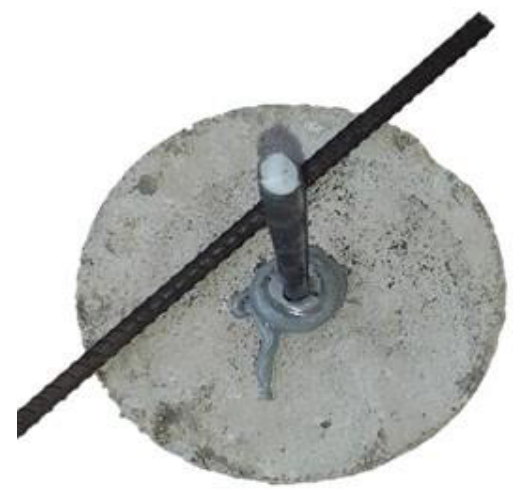

Figura 5. Falla del acero en anclaje estructural posinstalado Fuente: elaboración propia 


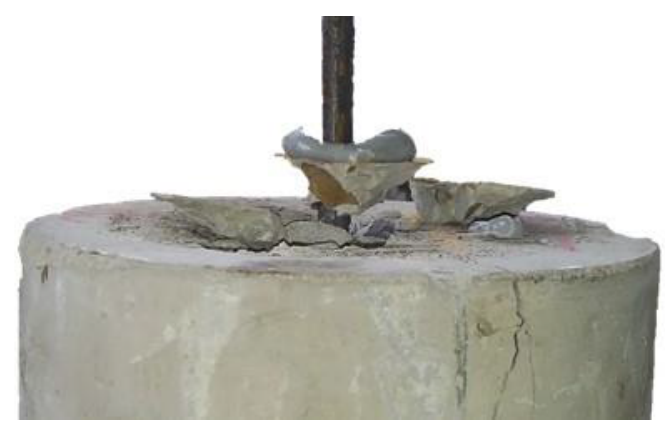

Figura 6. Falla por arrancamiento en anclaje estructural posinstalado Fuente: elaboración propia

En cuanto al análisis cuantitativo, a partir de la figura 7 se puede observar que para un mismo diámetro de perforación de $12,7 \mathrm{~mm}(\varnothing \mathrm{M})$ y una longitud de embebido de 15 veces el diámetro de la barra (Lex15) se desarrolla el $130 \%$ de la fluencia del acero llegando a su carga de rotura o carga última. Por su parte, con el mismo diámetro de perforación (ØМ) pero longitud de embebido de diez veces el diámetro de la barra a anclar (Lex10), se logró desarrollar el 113\% de la fluencia del acero, pero no se llegó a la carga de rotura; y en el caso de los anclajes con diámetro de perforación (ØМ) y la longitud de embebido más baja, es decir cinco veces el diámetro de la barra de refuerzo (Lex5) solo se alcanzó a desarrollar el $56 \%$ de la carga de fluencia. Para el resto de combinaciones de diámetro de perforación-longitud de embebido, en los cuales la longitud de embebido fue diez veces el diámetro de la barra a anclar (Lex10) y la condición a variar era el diámetro de perforación, solo dos de los nueve anclajes fallados logró desarrollar la fluencia del acero.

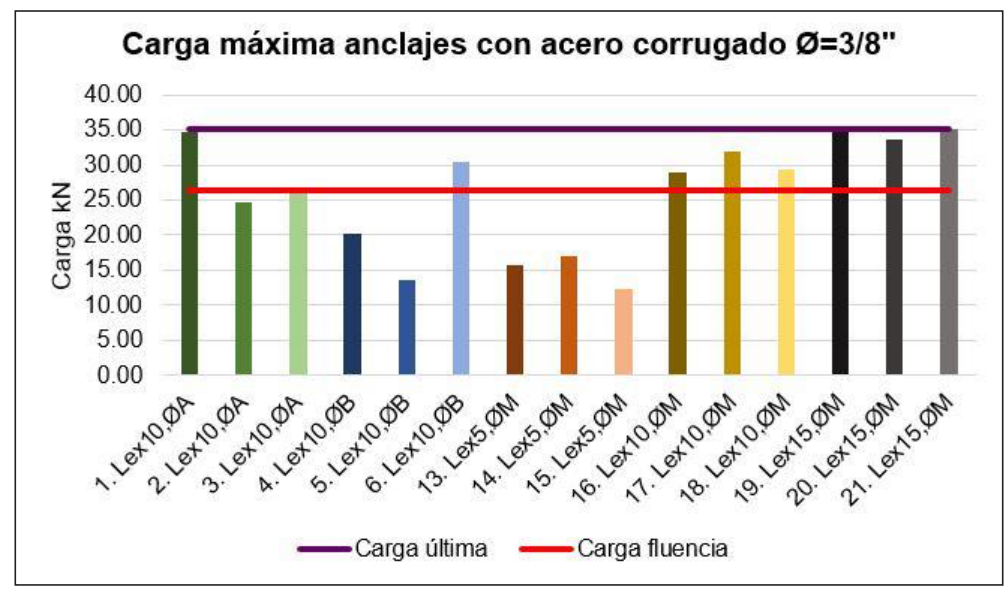

Figura 7. Carga máxima anclaje estructural post instalado con acero corrugado $\varnothing=3 / 8^{\prime \prime}$ Fuente: elaboración propia 
Respecto a la curva de resistencia de este tipo de anclajes, al analizar la figura 8 se observa claramente la semejanza entre la curva del acero y las curvas de los anclajes con mayor longitud de embebido (Lex15) dado que en estas se pueden diferenciar las zonas elástica, plástica y las cargas de fluencia y última. Asimismo, es evidente que en las curvas de resistencia correspondientes a las demás combinaciones de diámetro de perforación-longitud de embebido, la mayoría no alcanza a desarrollar zona plástica y por ende pasan súbitamente de la zona elástica a la falla. Es claro que en este tipo de anclajes el desarrollo de la fluencia, y que la falla sea de tipo dúctil o frágil, está intrínsecamente relacionado con la longitud de embebido.

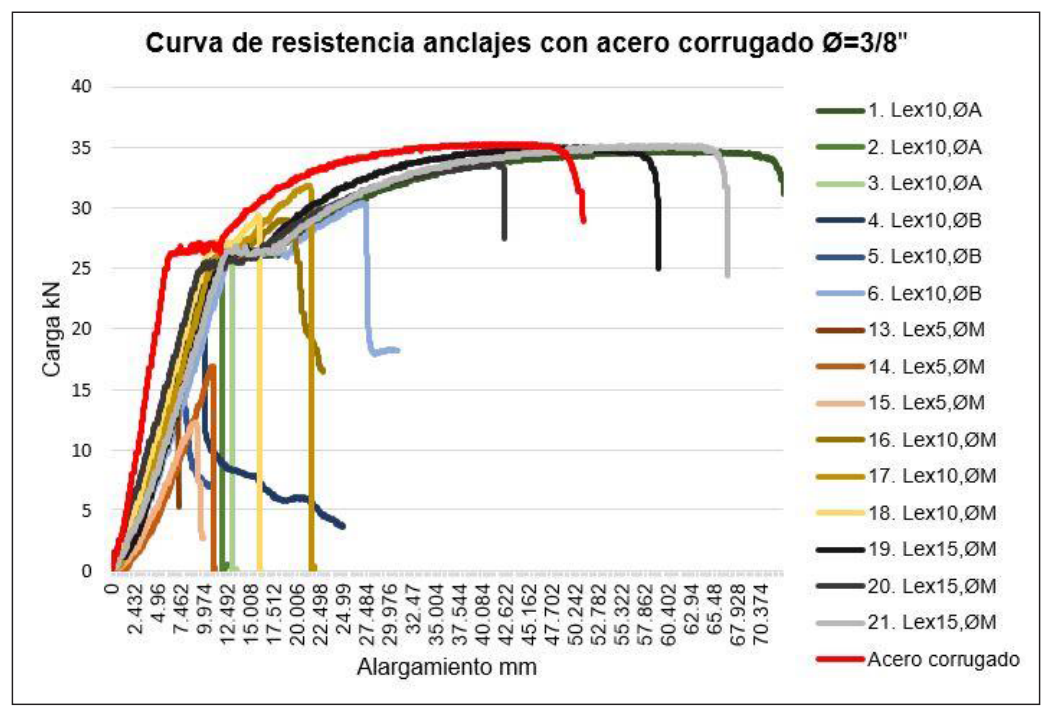

Figura 8. Curva de resistencia anclaje estructural posinstalado con acero corrugado $\varnothing=3 / 8^{\prime \prime}$ Fuente: elaboración propia

Por otra parte, respecto al grupo de anclajes estructurales posinstalados con grafil de diámetro $7 \mathrm{~mm}$, en la mayoría de estos la falla presentada fue de tipo arrancamiento como se exhibe en la figura 6. Asimismo, con base en la figura 9 se evidencia que solo uno de los quince anclajes fallados logró llegar a la carga de fluencia, mientras que a partir de la figura 10 se observa que el comportamiento de las curvas de resistencia de este tipo de anclajes no es lo suficientemente claro para ser analizado. Es evidente que se deben desarrollar más investigaciones respecto a este tipo de anclajes con barra de refuerzo de tipo grafil.

\section{CONCLUSIONES}

Considerando los materiales, diseño experimental y análisis empleados en este estudio se pueden obtener las conclusiones que a continuación se presentan. 
Los materiales empleados en esta investigación son de uso común en el sector construcción, especialmente el concreto [18] seleccionado. Esto permite generar trazabilidad en el desarrollo de nuevos estudios sobre esta temática, al igual que brinda la posibilidad de implementar los hallazgos encontrados en variedad de proyectos civiles como lo son aquellos de reforzamiento y restauración.

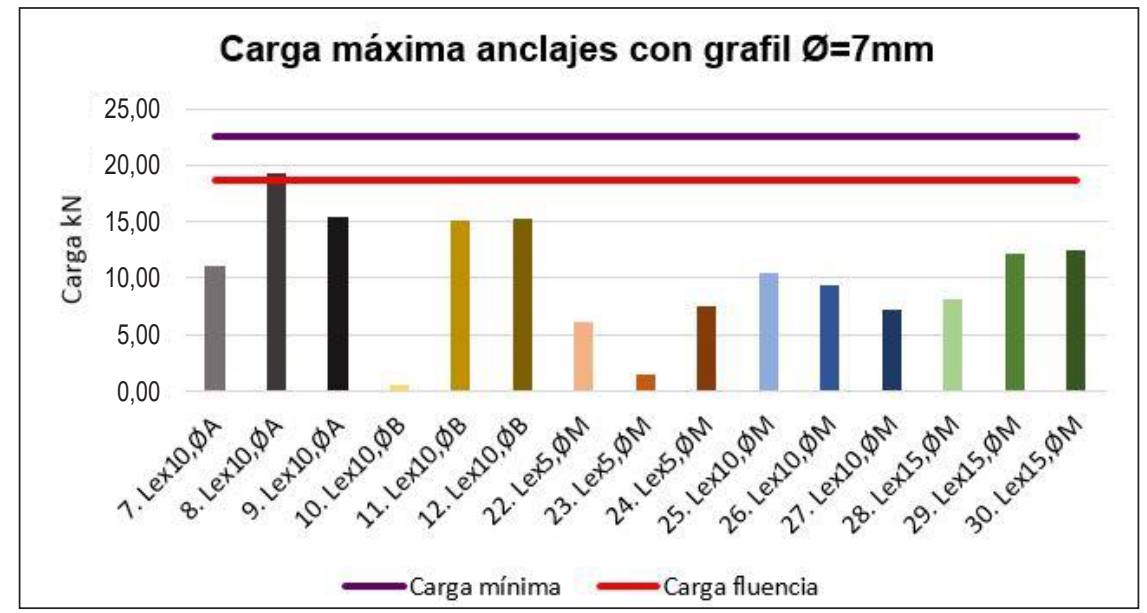

Figura 9. Carga máxima anclaje estructural posinstalado con grafil $\varnothing=7 \mathrm{~mm}$ Fuente: elaboración propia

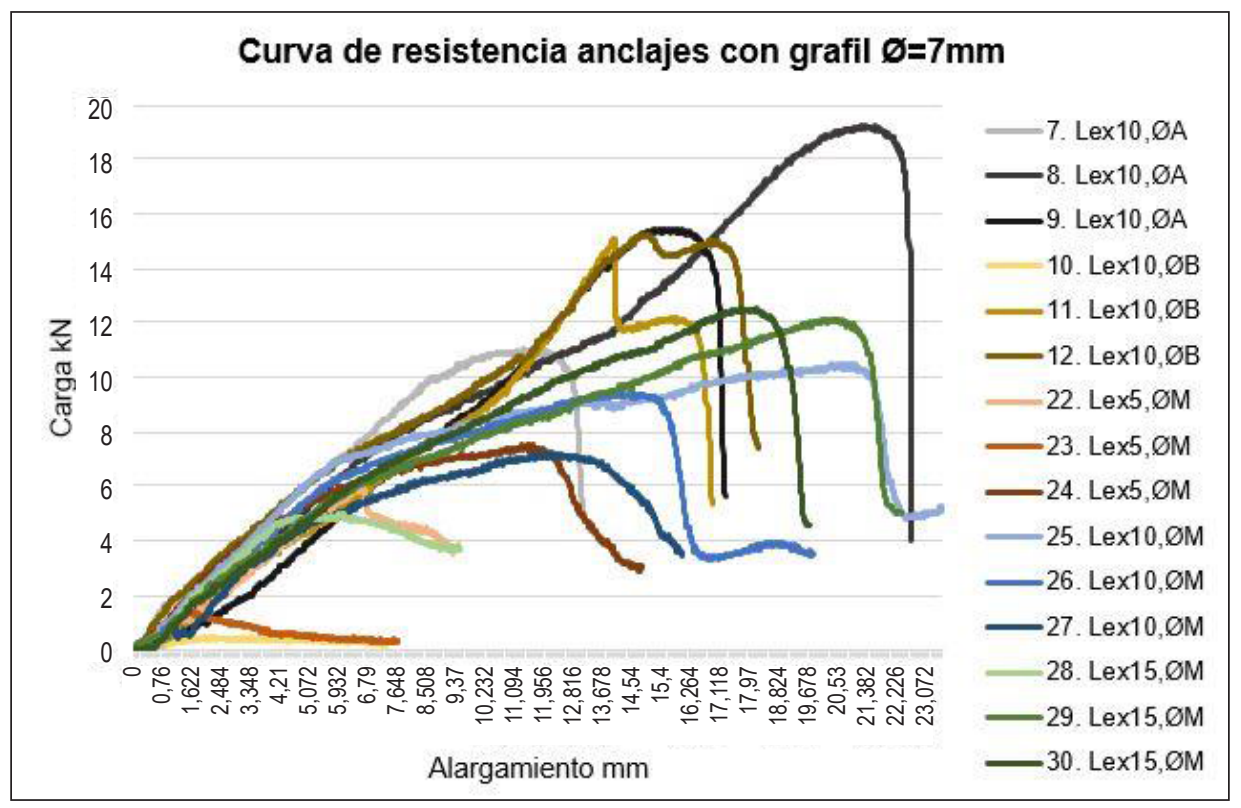

Figura 10. Curva de resistencia anclaje estructural post instalado con grafil $\varnothing=7 \mathrm{~mm}$ Fuente: elaboración propia 
La longitud de embebido ha sido una variable ampliamente estudiada en los anclajes estructurales posinstalados dada su incidencia en su comportamiento mecánico, y tal como se comprobó en esta investigación, esta variable es más determinante en el desempeño del anclaje que el diámetro de perforación.

Dados los resultados en los anclajes estructurales posinstalados con grafiles, se estableció la hipótesis de que la estructura de la corruga en este tipo de refuerzo no permite desarrollar suficiente adherencia en la interfaz refuerzo-adhesivo-concreto, razón por la cual la falla primaria de estos anclajes fue de tipo arrancamiento y su resistencia máxima no alcanza a desarrollar la fluencia de la barra. Es pertinente continuar investigando los anclajes estructurales posinstalados con adhesivo epóxico, específicamente anclajes con barra de refuerzo de tipo grafil.

Se estableció que, en los ensayos con barras de acero corrugado con anclajes en los que se usó longitud de embebido de cinco veces el diámetro de la barra (Lex5), la resistencia efectiva a tracción desarrolla hasta un $56 \%$ de la carga máxima de fluencia del acero de refuerzo, por lo cual, esta longitud de embebido se descarta y no se recomienda su implementación.

Los resultados de las cargas máximas de los sistemas de anclaje con acero corrugado evidencian que, al usar una longitud de embebido de 10 veces el diámetro de la barra (Lex10), se puede desarrollar la fluencia del acero de refuerzo. La falla primaria obtenida para esta longitud de embebido se caracteriza por la rotura del concreto.

Al aumentar la longitud de embebido a 15 veces el diámetro de la barra (Lex15) se alcanza a desarrollar hasta un $130 \%$ de la fluencia del acero, llegando incluso a la rotura de la barra. La falla primaria obtenida para esta longitud de embebido es de tipo dúctil, dado que el acero controla en su totalidad la capacidad del anclaje, excluyendo así las fallas en el concreto. Por lo anterior, esta longitud de embebido es la que se recomienda para la implementación de anclajes posinstalados.

\section{REFERENCIAS}

[1] República de Colombia. Ministerio de Ambiente, Vivienda y Desarrollo Territorial, Reglamento Colombiano de Construcción Sismo Resistente Nsr-10, Asociación Colombiana de Ingeniería Sísmica, 2010.

[2] J. Rendón, “Los anclajes adhesivos en la construcción”, Noticreto, vol. 126, pp. 43-47, 2014.

[3] Committee ACI 503, Use of epoxy compounds with concrete, ACI 503 R-93, American Concrete Institute, 1998.

[4] M. Gesoğlu et al., "Modeling and analysis of the shear capacity of adhesive anchors postinstalled into uncracked concrete," Composites: Part B: Engineering, vol. 60, pp. 716-724, 2014. 
[5] M. Obataa et al., "The failure mechanism and the pull-out strength of a bond-type anchor near a free edge," Mechanics of Materiales, vol. 28, pp. 113-122, 1998.

[6] P. Upadhyaya, y S. Kumar, "Pull-out capacity of adhesive anchors: An analytical solution," International Journal of Adhesion and Adhesives, vol. 60, pp.54-62, 2015.

[7] C. Ózlem et al., "Shear strength of epoxy anchors embedded into low strength concrete," Construction and Building Materials, vol. 38, pp. 723-730, 2012.

[8] S. Yang et al., "Theoretical analysis on pullout of anchor from anchor-mortar-concrete anchorage system," Engineering Fracture Mechanics, vol. 75, pp. 961-985, 2008.

[9] A. Çolak, "Parametric study of factors affecting the pull-out strength of steel rods bonded into precast concrete panels," International Journal of Adhesion and Adhesives, vol. 21, pp. 487-493, 2001.

[10] Y. M. Ruda Arias y D.F. Páez Moreno “Anclajes posinstalados en concreto de alta resistencia,” Ciencia e Ingeniería Neogranadina, vol. 27, N. ${ }^{\circ}$ 2, pp. 137-156, 2017.

[11] M. McVay, et al., "Pullout simulation of post installed chemically bonded anchors," Journal Structural Engineering, Vol.122, pp. 1016-1024, 1996.

[12] L. Contrafatto, y R. Cosenza, "Behavior of post-installed adhesive anchors in natural stone," Construction and Building Materiales, vol. 68, pp. 355-369, 2014.

[13] F. Puigvert et al., "Static analysis of adhesively bonded anchorages for CFRP tendons," Construction and Building Material, vol. 61, pp. 206-215, 2014.

[14] B. Gerber and M. Ekenel, "Building Code Requirements for Inspection of Adhesive Anchors in Concrete", Journal of Construction Enigneering and Management, vol. 139, 2013.

[15] Post-Installed Reinforcing Bar Guide, Hilti Group, 2016.

[16] Committee ACI 318-11, Building Code Requirements for Structural Concrete and Commentary, American Concrete Institute, 2011.

[17] Sika Colombia S.A., "Hoja técnica Sikadur AnchorFix-4," [En línea], acceso 14 de abril, 2015; Disponible: http://col.sika.com/es/rehabilitacion-y-reforzamiento/rehabilitarreforzar/02a005/02a005sa01.html

[18] M. Lascarro, “Indicadores en concreto,” [En línea], acceso 14 de abril, 2015; Disponible: https://www.construccionlatinoamericana.com/indicadores-en-concreto/128416.article

[19] Icontec, Concretos. Ensayo resistencia a la compresión de especímenes cilíndricos de concreto, NTC 673, Instituto Colombiano de Normas Técnicas y Certificación Icontec, 2012.

[20] Icontec, Siderurgia. Ensayo de tracción para materiales metálicos. Método de ensayo a temperatura ambiente, NTC 2, Instituto Colombiano de Normas Técnicas y Certificación Icontec, 1995.

[21] ASTM International, ASTM E488/488M-10 Standard Test Methods for Strength of Anchors in Concrete Elements, ASTM INTERNATIONAL, 2014.

[22] Committee ACI 355, Report on Anchorage to Concrete, ACI 355 1R-91 (Reapproved 1997), American Concrete Institute, 1997. 